Research Article

\title{
Deep Learning Based Coronary Angiography in Diagnosis of Myocardial Ischemia
}

\author{
Yiwen Shu 1 and Xiwen Wu \\ Department of Cardiology, The First People's Hospital of Huaihua, Huaihua 418000, Hunan, China \\ Correspondence should be addressed to Xiwen Wu; wanglu98@sues.edu.cn
}

Received 9 July 2021; Revised 14 August 2021; Accepted 16 August 2021; Published 6 September 2021

Academic Editor: Gustavo Ramirez

Copyright (C) 2021 Yiwen Shu and Xiwen Wu. This is an open access article distributed under the Creative Commons Attribution License, which permits unrestricted use, distribution, and reproduction in any medium, provided the original work is properly cited.

Objective. This study was to explore the diagnostic effect of the coronary angiography (CAG) based on the fully convolutional neural network (FCNN) algorithm for patients with coronary heart disease (CHD) and suspected (not diagnosed) myocardial ischemia. Methods. In this study, 150 patients with undiagnosed CHD with myocardial ischemia in hospital were selected as the research objects. They were divided into an observation group and a control group by random number method. The patients in observation group were examined with CAG with the assistance of convolutional neural network (CNN) algorithm, while patients in the control group received conventional CAG. Results. The Dice coefficient of the segmentation effect evaluation index was 0.89 , which showed that the image processing effect of the algorithm was good. There was no statistical difference in positive rates of single/double-vessel lesions between the two groups $(P>0.05)$, and the positive rates of multivessel lesions and total lesions in the observation group were higher than those in the control group, showing statistically obvious difference $(P<0.05)$. The examination sensitivity, specificity, accuracy, and Kappa value of the observation group were $-90.9 \%,-60 \%,-82.7 \%$, and -0.72 , which were all higher in contrast to those of the control group. The proportion of positive myocardial ischemia and coronary artery stenosis (CAS) $(82 \%)$ was higher than other cases $(18 \%)$, and the comparison was statistically significant $(P<0.05)$. Conclusion. CAG based on the deep learning algorithm showed a good detection effect and can better display the coronary lesions and reflect the good development prospects of deep learning technology in medical imaging.

\section{Introduction}

Myocardial ischemia is a common complication in the development of coronary atherosclerotic heart disease (also known as coronary heart disease (CHD)). On the contrary, $\mathrm{CHD}$ is also the main cause of myocardial ischemia [1,2]. Myocardial ischemia is a pathological state, and its pathogenesis is mainly that insufficient blood flow leads to a decrease in oxygen supply to the heart, which causes abnormal metabolism of myocardial cells and the heart cannot maintain normal functions [3]. Myocardial ischemia can also cause various serious adverse reactions such as sudden death and myocardial infarction. At this stage, the incidence of myocardial ischemia in China is increasing year by year. The main affected population is middle-aged and elderly people. However, some young people (20 30 years old) begin to show symptoms of myocardial ischemia. Therefore, it has to pay attention to the diagnosis and treatment of the disease-early diagnosis, early treatment, and prevention of deterioration [4-6].

Coronary angiography (CAG) is the main index for clinical detection and diagnosis of coronary atherosclerotic lesions, and it can directly show the degree of stenosis of the main coronary artery and its branches [7, 8]. However, the detection of CAG still has shortcomings. For example, the doctor's assessment of the relationship between CAS and myocardial ischemia produces errors due to the limitation of the irradiation angle and body position [9]. The geometric structure analysis of coronary blood vessels is very important in the diagnosis of related diseases. Therefore, people have proposed that it is necessary to study the image segmentation algorithm of coronary angiography to make reliable 
judgments in the clinical application of CAG. In addition, automatic segmentation of the vascular tree sequence can effectively reduce the doctor's misjudgment due to subjective experience and fatigue [10-12]. With the development of computer vision field, convolutional neural network (CNN) has brought a revolutionary subversion to large-scale image processing, and related methods have also made great progress in the field of medical image processing [13]. The segmentation of medical images belongs to the category of semantic segmentation in deep learning, which essentially classifies every pixel. In recent years, semantic segmentation has made great progress [14-16]. For instance, fully convolutional neural networks (FCNN) are a pioneering change. The algorithm removes the fully connected layer at the end, and subsequent semantic segmentation networks are improvements based on this structure $[17,18]$. In order to make the effect of CAG more favorable for diagnosis, some experts proposed the combination of FCN and CAG technology, and good results were obtained after research.

Therefore, the CAD based on the FCNN was adopted to examine and diagnose patients with $\mathrm{CHD}$ and suspected (not diagnosed) myocardial ischemia to analyze the relationship between myocardial ischemia and CHD and explore its diagnostic effect and the guiding significance for the treatment of myocardial ischemia. It was hoped that more treatment methods can be provided for all patients suffering from myocardial ischemia, and to further improve the timeliness of diagnosis and treatment of their diseases.

\section{Methods}

2.1. Blood Vessel Segmentation Method under CAD Sequence of FCNN. Firstly, the CAD sequence images had to be preprocessed to make the model more generalized and the network model obtained by training better, so a lot of supportive data were needed, but it was difficult to collect enough training samples. Therefore, a good foundation was created for the training of the model by enhancing the existing data. There were two main enhancement methods adopted in this study: one was data enhancement and the other was contrast enhancement.

Data enhancement mainly was realized through mirror symmetry and image rotation, so that the enhanced effect can be closer to the real data. The effect is shown in Figures 1 and 2 .

Contrast enhancement was achieved by changing the grayscale range of the image through the grayscale transformation method. The grayscale value range had to be increased to increase the contrast. It meant that a smaller gray scale range was mapped to a larger range through the range stretching operation. The general expression of the gray mapping function was as follows:

$$
f=\frac{a-b}{c-d}(e-d)+b .
$$

In the above equation, $[\mathrm{d}, \mathrm{c}]$ was the initial value range. The initial image is shown in Figure 3(a); $[\mathrm{b}, \mathrm{a}]$ was the stretched value range (as shown in Figure $3(\mathrm{~b})$ ), $e$

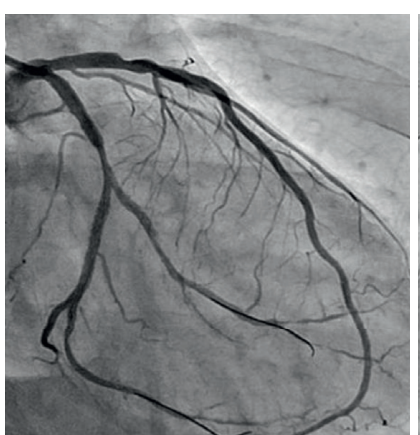

Original picture

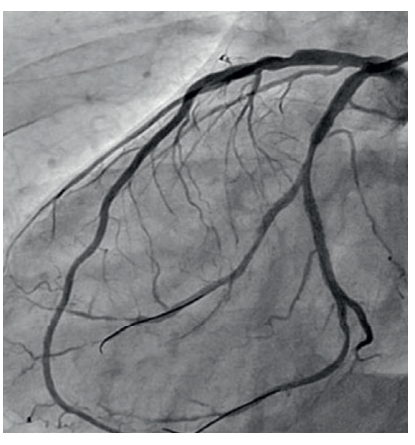

Mirror symmetry
FIGURE 1: Enhancement effect of image using mirror symmetry.

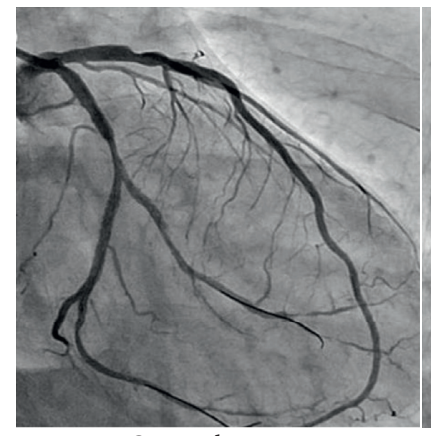

Original picture

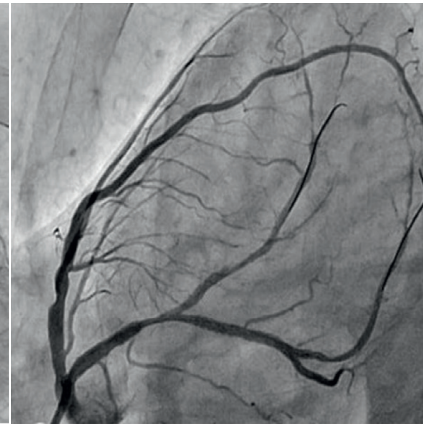

Rotate $90^{\circ}$
Figure 2: The enhancement effect of the image rotated by $90^{\circ}$.

represented the original image matrix, and $f$ referred to the stretched image matrix.

After the preprocessing was completed, the segmentation design of CAD sequence based on the FCNN could be started. Convolutional neural network $(\mathrm{CNN})$ can be divided into input layer, convolutional layer, pooling layer, fully connected layer, activation function, and output. The FCNN removed the fully connected layer and performed deconvolution calculation on the output of the last convolution layer to obtain a feature map with the same size as the original image.

The convolutional layer can be expressed as follows:

$$
\begin{aligned}
q(i, i) & =(\mathrm{WW} * \mathrm{RR})(i i, j j) \\
& =\sum_{m m} \sum_{n n} w w(i i-m m, j j-n n) r r(m m, n n) .
\end{aligned}
$$

In (2) above, $W$ was the input signal, $R$ was the convolution kernel, and $q$ represented the output signal. The above equation can also be changed to the following equation for easier calculation:

$$
\begin{aligned}
q(i, i) & =(W W * R R)(i i, j j) \\
& =\sum_{m m} \sum_{n n} w w(i i+m m, j j+n n) r r(m m, n n) .
\end{aligned}
$$

The image segmentation in this study can be regarded as pixel-level classification, and the commonly used loss function was cross entropy. Cross entropy determined the 


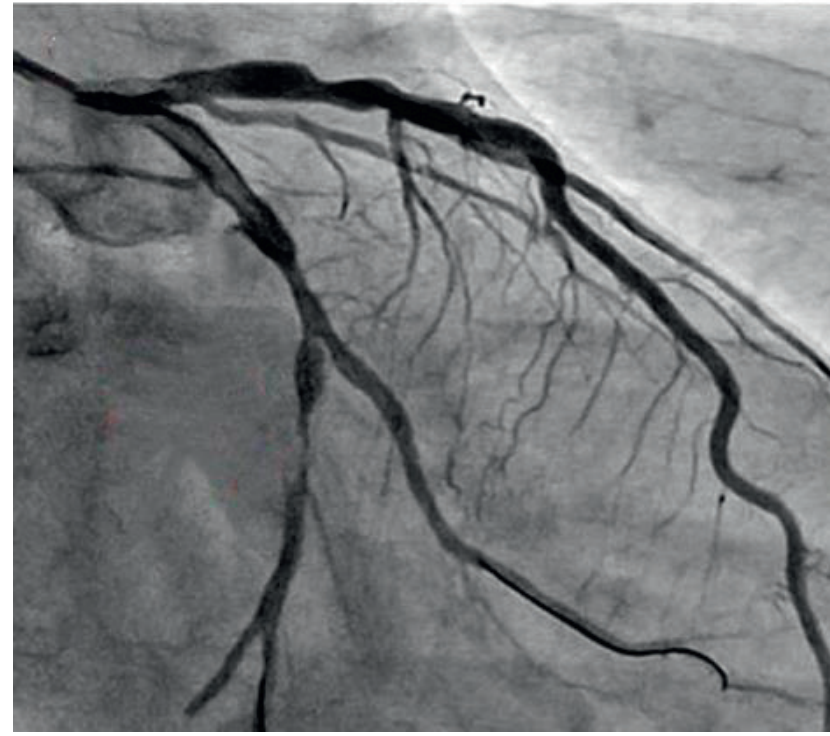

(a)

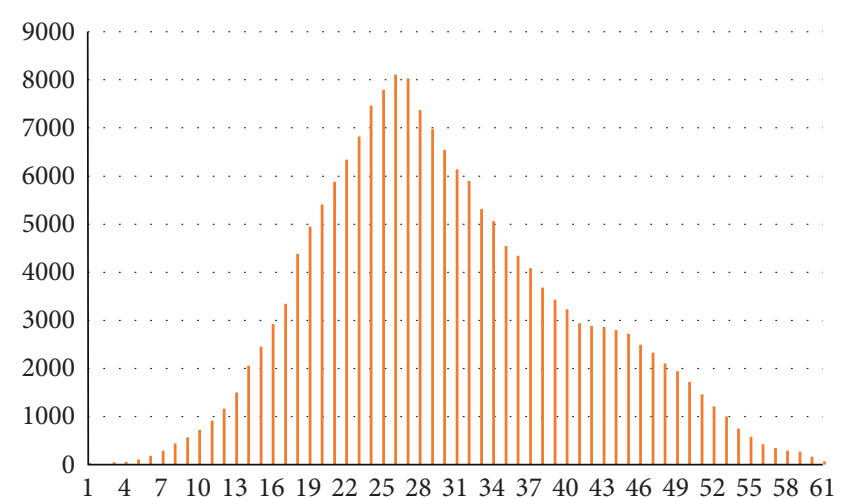

(b)

Figure 3: The gray value range of the image. (a) Original picture. (b) Gray statistic chart.

relationship between two different data distributions, and the expression was as follows:

$$
L=-[x \log \bar{x}+(l-x) \log (l-\bar{x})] .
$$

In the above equation, $x$ referred to the true category of the data and $\bar{x}$ represented the predicted category of the data.
However, the result of this expression was insufficient. In this study, the weights of positive and negative samples were improved, and the loss function of coronary angiography images was defined as adaptive class-balanced cross entropy. The new expression was as follows:

$$
\begin{aligned}
L(Q) & =-\sum_{j} \beta y_{i} \log W\left(y_{i}=l|R ; Q|\right)+(l-\beta)\left(l-y_{i}\right) \log W\left(y_{i}=0|R ; Q|\right) \\
& =-\beta \sum_{j \in Y_{+}} \log W\left(y_{i}=l|R ; Q|\right)-(l-\beta) \sum_{j \in Y_{-}} \log W\left(y_{i}=0|R ; Q|\right) .
\end{aligned}
$$

In the equation above, $Q$ referred to training parameter of CNN, $R$ represented the input image, $j \in(0,1)$ was the pixel label, $W$ represented the predicted probability of each pixel network output, and $\beta$ was the weight of positive and negative samples, which could be defined as

$$
\beta=\frac{X_{-}}{X} .
$$

In the equation above, $X$ - referred to the negative sample size, and $X$ represented the total sample size. The weight of the positive sample loss function was higher, and the weight of the negative sample was reduced, which can effectively solve the imbalance among samples.

For CAD image segmentation in this study was realized using conditional random field (CRF) to construct the energy function of the conditional random field on the CAD sequence images. Then the CRF-based image semantic segmentation problem was to predict the corresponding category of the pixel Xi by observing the variables and their characteristics. The Gibbs distribution corresponding to the conditional random field $(X, I)$ was defined as follows:

$$
G(X \mid I)=\frac{1}{Z(I)} \exp \left(-\sum_{c \in c_{s}} \phi_{s}\left(X_{c} \mid I\right)\right) .
$$

Then, for image $T, \mathrm{~S}$ was the image model on $X$, I represented $\mathrm{N}$ inputs, $\mathrm{C}$ represented the maximum subgraph, $Z$ was the normalization factor, $F$ meant the potential function, and CS referred to the set of all the largest subgraphs of graph S. E was set to be the connection between every two pixels, and then the energy of a Gibbs label $x \varepsilon L$ was written as follows:

$$
E(X \mid I)=\sum_{c \in c_{s}} \phi_{s}\left(X_{c} \mid I\right) .
$$

The maximum a posteriori estimate (MAP) of the CRF was given in the following equation:

$$
X^{*}=\operatorname{argmax}_{x \in L} W(x \mid I) .
$$


In a fully connected binary CRF, $S$ referred to the set of all unary and binary largest subgraphs on the complete graph on $X$, and then the corresponding Gibbs energy was shown as follows:

$$
E(X)=\sum_{z} \alpha_{i}\left(x_{z}\right)+\sum_{z<v} \alpha_{j}\left(x_{z}, x_{v}\right)
$$

The value range of $z$ and $v$ in the above equation was [ 1 , $\mathrm{N}], a_{i}$ was one-variable potential function, and $a_{j}$ was twovariable potential function.

The specific description of the above two functions was as follows, which was a unary potential function:

$$
\alpha_{i}\left(x_{z}\right)=-\sum_{z} \log P\left(x_{z}\right) .
$$

$P\left(X_{z}\right)$ in the equation above referred to the probability of each pixel predicting the label after passing through the FCNN. According to the equation, the function was a description of the predicted probability of a single pixel label. The binary potential function was written as follows:

$$
\alpha_{j}\left(x_{z}, x_{v}\right)=\mu\left(x_{z}, x_{v}\right) \sum_{m-1}^{k} w^{(m)} k^{(m)}\left(x_{z}, x_{v}\right) .
$$

In the above equation, $\mu\left(x_{z}, x_{v}\right)$ was a judgment function, which determined whether the binary potential function can be calculated as a potential function among different nodes, $w^{(m)}$ was a linear combination weight, $k^{(m)}\left(x_{z}, x_{v}\right)$ referred to the Gaussian kernel function, and $\left(x_{z}, x_{v}\right)$ represented the feature vector of pixels $z$ and $v$.

In image segmentation tasks, the kernel function often takes contrast-sensitive two-kernel potential functions. These two potential functions were defined by the color vector $h$ and position $b$, as follows:

$$
\begin{aligned}
k\left(x_{z}, x_{v}\right)= & w^{(1)} \exp \left(-\frac{\left|b_{z}-b_{v}\right|^{2}}{2 \theta_{\alpha}^{2}}-\frac{\left|h_{z}-h_{v}\right|^{2}}{2 \theta_{\beta}^{2}}\right) \\
& +w^{(2)} \exp \left(-\frac{\left|b_{z}-b_{v}\right|^{2}}{2 \theta_{\chi}^{2}}\right) .
\end{aligned}
$$

In the above equation, $w^{(1)} \exp \left(-\left(\left|b_{z}-b_{v}\right|^{2} / 2 \theta_{\alpha}^{2}\right)-\left(\left|h_{z}-h_{v}\right|^{2} / 2 \theta_{\beta}^{2}\right)\right)$ was the first potential function, which was called the appearance kernel; its definition came from the guess that the observed pixels of similar colors were likely to be of the same type; and the similarity was controlled by the parameters $\theta \alpha$ and $\theta \beta$. $w^{(2)} \exp \left(-\left(\left|b_{z}-b_{v}\right|^{2} / 2 \theta_{\chi}^{2}\right)\right)$ was the second number, which was called a smooth kernel, and its function was to eliminate small isolated areas; the similarity of isolated areas was controlled by the parameter $\theta \chi$.

In the actual training process, the fully connected CRF is added to the last layer of the FCNN for training. It took the final output feature map with classification probabilities in the full convolution as input to establish the fully connected CRF (Figure 4).

In the evaluation of segmentation results, the Dice coefficient was adopted to reflect the closeness between the result of the image pixel-level classification and the actual result, which could be expressed as (14). In the equation, $\Upsilon$ and $\vartheta$ represented two samples, and the result range of Dice was in $[0,1]$. The closer the range to 1 , the higher the sample similarity, indicating that the predicted result was more accurate.

$$
\text { Dice }=\frac{2|\Upsilon \cap \vartheta|}{|\Upsilon|+|\vartheta|}
$$

After calculation and analysis, the Dice coefficient was 0.89 based on the fully convolutional neural network model in the segmentation of coronary angiography sequence images, indicating that the algorithm had good image processing effects and can be used in clinical research applications.

2.2. Research Objects. 150 undiagnosed CHD patients with myocardial ischemia who were admitted to the hospital from July 2018 to January 2021 were selected as research objects, including 81 male patients and 69 female patients. The age was $46-79$ years, with the average age of $60.15 \pm 12.15$ years; and the course of disease was 3-12 months, with the average course of disease of $6.06 \pm 1.54$ months. They were divided into an observation group and a control group using random number method, with 75 cases in each group. The patients in observation group received CAD based on the FCNN for disease detection, and the patients in control group used conventional CAD methods for inspection and diagnosis. The results of clinical diagnosis, electrocardiogram (ECG), and other biochemical tests were used as the standard. The study had been reviewed and approved by the Medical Ethics Committee, and all patients had signed the informed consent forms.

The inclusion criteria were defined as follows: patients whose coronary artery examination revealed that the diameter of the blood vessel was above $2.25 \mathrm{~mm}$ and the CAS was $50 \% \sim 70 \%$ (critical lesion); patients with symptoms of chest pain and chest tightness without persistent atrial fibrillation or cardiomyopathy, heart failure, conduction block, and preexcitation syndrome; and patients who could receive the tolerable inspection.

The exclusion criteria were defined as follows: patients whose coronary artery imaging showed severe artifacts, dislocation, noise, and calcified plaque; patients whose vessel diameter $<2.5 \mathrm{~mm}$ or tortuous and who were unable to carry out CAG detection; patients combined with severe liver and kidney insufficiency, heart failure, left ventricular hypertrophy, or a history of coronary artery bypass grafting.

2.3. CAG Examination. All patients were examined by the same physician with high qualifications and rich operating experience, and the images were reviewed by two doctors. Before CAG examination, preoperative preparations were required, including preparation of equipment, medicines, and staff in the examination room; preoperative signing of an informed consent; completion of preoperative inspections of various indicators, skin preparation, iodine allergy 


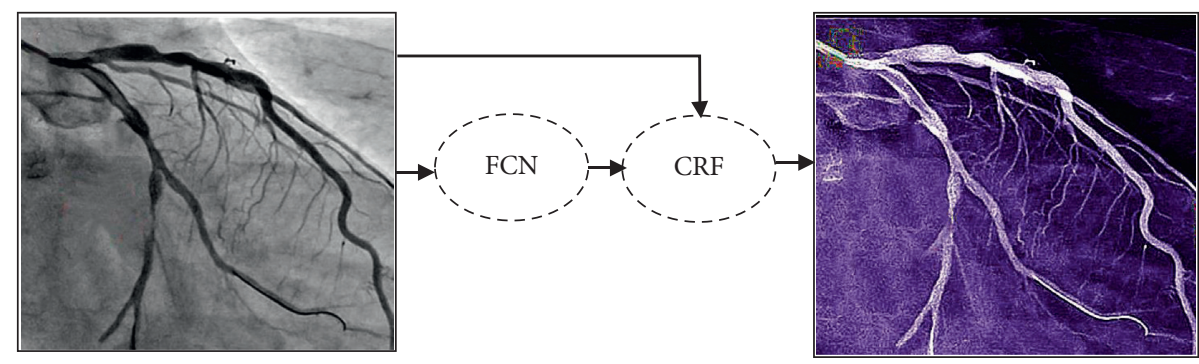

FIGURE 4: Image processing process.

test, and indwelling needle puncture. In addition, $200 \mu \mathrm{g}$ nitroglycerin should be given through the coronary artery to avoid coronary artery spasm. Most of the acupuncture approaches for CAD was the limb arteries, of which percutaneous puncture of the radial artery was the most commonly used. The appropriate approach was selected according to the patient's own situation. Afterward, the $6 \mathrm{~F}$ radiography catheter was used as a reference, and the software of the interventional radiography of the radiography machine was used to detect the coronary arteries in multiple directions, and to understand the degree of stenosis of the target diseased artery and the length of the disease.

2.4. Observation Indicators. The general data between the two groups of patients were compared, including gender, age, smoking history, history of diabetes, and blood lipid levels.

The positive lesion rates between the two groups of patients were compared. CAS $>50 \%$ were defined as positive lesions, including left anterior descending artery, left circumflex artery, and right coronary artery. The vascular lesions of more than two vessels were multivessel lesions. The relationship between CHD and myocardial ischemia in the two groups of patients were analyzed and compared.

The diagnostic results of patients in different groups were compared, and the sensitivity, specificity, and accuracy of the two examination methods were analyzed and compared. The specific calculation methods of sensitivity, specificity, and accuracy were as follows: take the parameter settings in Table 1 as an example.

Then, the specific calculation methods of sensitivity, specificity, and accuracy were as follows:

$$
\begin{aligned}
& \text { sensitivity }=\frac{a}{A 1} \times 100 \%, \\
& \text { specificity }=\frac{d}{B 1} \times 100 \%, \\
& \text { accuracy }=\frac{(a+d)}{E} \times 100 \% .
\end{aligned}
$$

2.5. Statistical Analysis. The SPSS22.0 statistical software system was adopted for data entry, sorting, and statistical analysis. The comparison of enumeration data adopted the $\chi^{2}$ test; the comparison of measurement data adopted the $t$ -
TABLe 1: Parameter settings.

\begin{tabular}{lccc}
\hline & Positive & Negative & Total \\
\hline Positive & True positive (a) & False positive (b) & C1 \\
Negative & False negative (c) & True negative (d) & D1 \\
Total & A1 & B1 & E \\
\hline
\end{tabular}

test; and the comparison of multiple sample means adopted the analysis of variance. The least significant difference (LSD) method was used when the variance was uniform, and the Dunnett T3 method was adopted when the variance was uneven. $P<0.05$ meant the difference was statistically different. Kappa test was performed on the consistency between the results of the two groups of CAD examination and the comprehensive diagnosis. When Kappa $>0.75$, the consistency between the two was strong; when $0.4 \leq$ Kappa $<0.75$, the consistency between the two was general; and when Kappa $<0.4$, the consistency between the two was poor.

\section{Results}

3.1. Dice. After calculation and analysis, the Dice coefficient obtained from the segmentation of coronary angiography sequence images based on the full convolution neural network model was 0.89 , which showed that the image processing effect of the algorithm was good and could be used in clinical research and application.

3.2. Comparison on General Data of Patients. According to statistics, the gender distribution of the two groups was as follows: males: $48.1 \%$ in the control group and $51.9 \%$ in the observation group, female: $51.5 \%$ in the control group and $48.5 \%$ in the observation group; age was as follows: $62.18 \pm 6.12$ years in the control group and $59.09 \pm 6.25$ years in the observation group; smoking history was as follows: 45 cases in the control group and 39 cases in the observation group; and the history of diabetes and blood lipid levels are shown in Table 2. Through analysis and comparison, it was found that the comparison was not statistically significant $(P>0.05)$.

3.3. Comparison on CAS Positive Rate. Table 3 shows the results of CAD examination of the two groups of patients. In the observation group, there were 24 single-vessel lesions with a positive rate of $32.00 \%$; 16 double-vessel lesions with a positive rate of $21.33 \%$; and 10 multivessel lesions with a 
TABLE 2: Comparison on general data of patients in two groups.

\begin{tabular}{lccc}
\hline & Group & Observation group $(n=75)$ & Control group $(n=75)$ \\
\hline Gender & Males $(n=81)$ & 42 & 39 \\
Age (years) & Females $(n=69)$ & 32 & 34 \\
Smoking history (cases) & & $59.09 \pm 6.25$ & $62.18 \pm 6.12$ \\
Diabetes history (cases) & & 45 & 39 \\
& Total cholesterol & 17 & 19 \\
Cholesterol (mmol/L) & High density lipoprotein (HDL) cholesterol & $1.15 \pm 0.45$ & $4.01 \pm 0.41$ \\
Triacylglycerol (mmol/L) & Low density lipoprotein (LDL) cholesterol & $3.79 \pm 0.37$ & $1.03 \pm 0.12$ \\
& & $1.61 \pm 0.14$ & $3.99 \pm 0.26$ \\
\hline
\end{tabular}

TABLE 3: Statistics of the inspection results of the two groups of lesions.

\begin{tabular}{|c|c|c|c|c|}
\hline Group & Single-vessel lesions (cases) & Double-vessel lesions (cases) & Multivessel lesions (cases) & Total (cases) \\
\hline Observation group $(n=75)$ & 24 & 16 & 10 & 50 \\
\hline Control group $(n=75)$ & 20 & 14 & 4 & 38 \\
\hline
\end{tabular}

positive rate of $13.33 \%$. In the control group, there were 20 single-vessel lesions with a positive rate of $26.67 \%$; there were 14 cases of double-vessel lesions with a positive rate of 18.67\%; and there were 4 cases of multivessel lesions with a positive rate of $5.33 \%$. After comparison, there was no obvious statistical significance between the positive rates of single-vessel lesions and double-vessel lesions in the two groups $(P>0.05)$, while the comparison between the positive rates of multivessel lesions was statistically significant $(P<0.05)$ (as illustrated in Figure 5(a)). The statistical analysis (Figure 5(b)) suggested that the total number of lesions in the observation group was 50 cases, and the total positive rate was $66.78 \%$; the total number of lesions in the control group was 38 cases, and the total positive rate was $50.67 \%$. The positive rate in the observation group was higher in contrast to the control group, showing statistically great difference $(P<0.05)$.

3.4. Comparison in Consistency of Comprehensive Diagnosis Results. Table 4 shows the statistical results of comprehensive diagnosis of the observation group and the experimental group. The comprehensive diagnosis was the "gold standard." The sensitivity, specificity, and accuracy in the examination results of observation group were $90.9 \%, 60 \%$, and $82.7 \%$, respectively. The sensitivity, specificity, and accuracy in the examination results of the control group were $67.8 \%, 52.6 \%$, and $64 \%$, respectively. After comparative analysis, it was found that the sensitivity, specificity, and accuracy of the examination results of the observation group were higher than those of the control group, showing statistically great difference $(P<0.05)$, as shown in Figure 6 . The consistent Kappa values in the observation group and the control group were 0.72 and 0.67 , respectively. Analysis suggested that the consistency with the comprehensive diagnosis of the observation group was strong, while that in the control group was generally consistent with the comprehensive diagnosis, showing statistically observable difference $(P<0.05)$. This suggested that the examination effect of the observation group was better than that of the control group.
3.5. The Relationship between CAS and Myocardial Ischemia. Table 5 shows the comprehensive diagnosis of the two groups of $\mathrm{CHD}$ patients with clinical manifestations of myocardial ischemia.

The proportion of positive diagnosis result of myocardial ischemia with CAS was $82 \%$. The proportion of nonstenosis was $10.7 \%$; the proportion of negative diagnosis result with stenosis was $3.3 \%$, and the proportion of nonstenosis was $4 \%$. After comparison, it was found that the proportion of CHD patients with positive myocardial ischemia and CAS (82\%) was much higher than the proportion of other conditions $(18 \%)$, and the comparison was statistically significant $(P<0.05)$, as shown in Figure 7 . This suggested that there was a great relationship between CAS and myocardial ischemia in CHD patients.

\section{Discussion}

Changes in blood pressure, aortic blood supply, blood viscosity, and coronary artery occlusion can all induce myocardial ischemia $[19,20]$. It is known from the above that the most important and common predisposing factor for myocardial ischemia is CHD. The results of this study showed that most patients with CHD diagnosed as positive for myocardial ischemia suffered from CAS, accounting for $82 \%$ of the total number. Such results suggested that the possibility of coronary artery occlusion caused by myocardial ischemia is extremely high. However, some research experts have used treadmill exercise test to analyze the correlation between CAS and myocardial ischemia, and 30 patients with stable angina pectoris were taken as the research objects. The results showed that exercise treadmill test had a high application value in the diagnosis of myocardial ischemia, and there was no positive correlation between CAS and myocardial ischemia [21, 22]. This is obviously different from the research results of this study, which may be related to the disease basis of the research subjects and the different research methods. Therefore, further comprehensive research and analysis is needed. In this study, the application effect of CAD based on deep learning was analyzed in the 


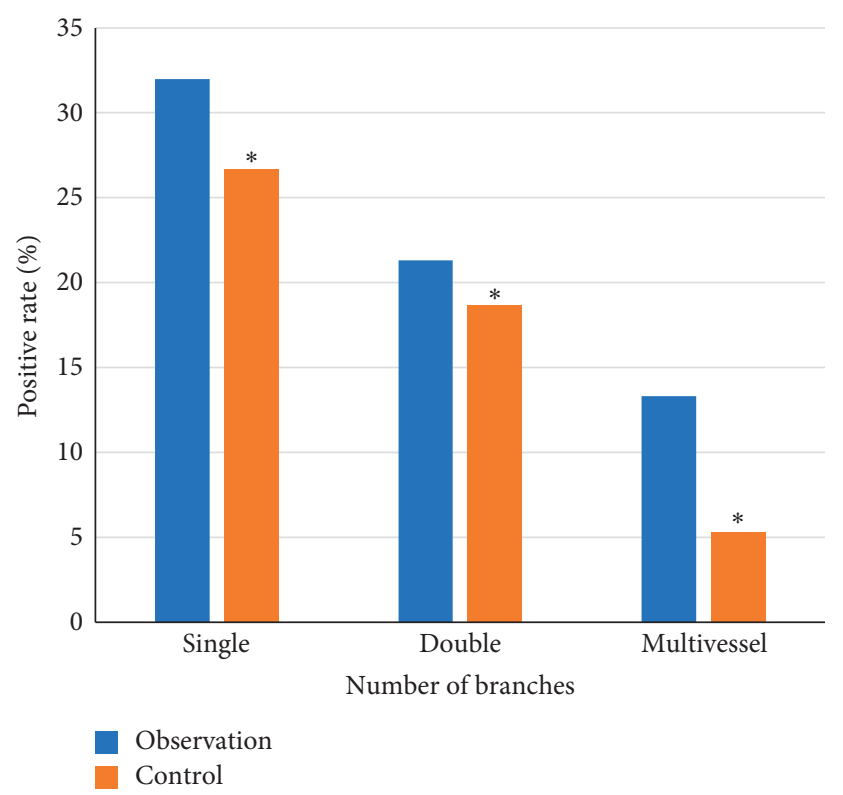

(a)

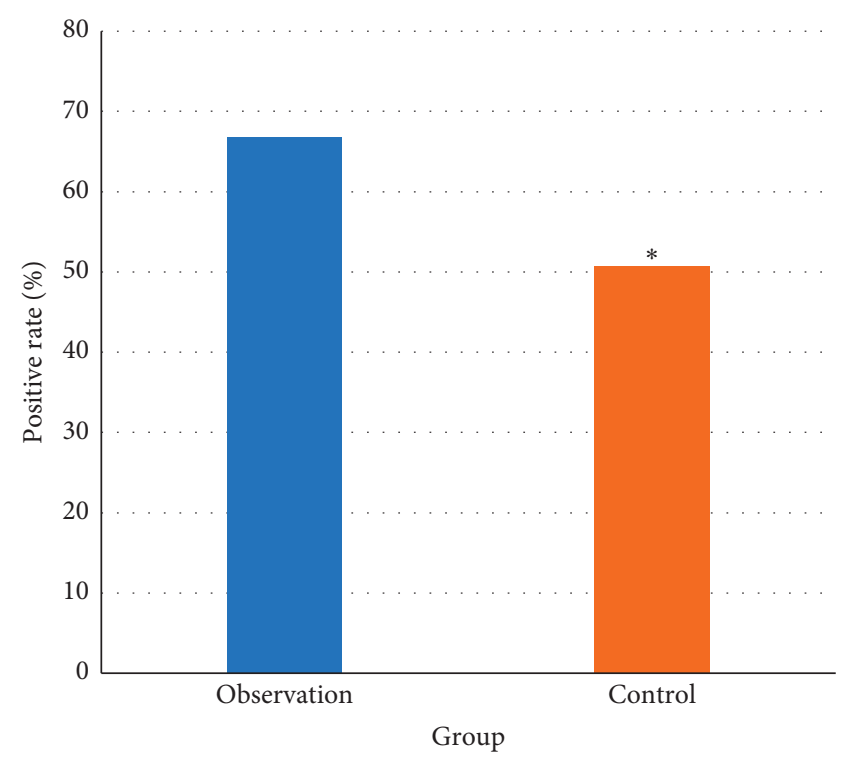

(b)

FIGURE 5: Comparison on positive rate of two groups. (a) The comparison on positive rate of different vessel lesions; (b) the comparison on positive rates of total lesions. $*$ denotes the difference between the two groups was statistically significant $(P<0.05)$.

TABLE 4: Statistics of the diagnosis results of the two examination methods.

\begin{tabular}{|c|c|c|c|c|}
\hline \multirow{2}{*}{\multicolumn{2}{|c|}{ Group }} & \multicolumn{2}{|c|}{ Comprehensive diagnosis } & \multirow{2}{*}{ Total } \\
\hline & & Positive & Negative & \\
\hline \multirow{2}{*}{ Observation group ( $n=75$} & Positive & 50 & 8 & 58 \\
\hline & Negative & 5 & 12 & 17 \\
\hline Total & & 55 & 20 & 75 \\
\hline \multirow{2}{*}{ Control group $(n=75)$} & Positive & 38 & 9 & 43 \\
\hline & Negative & 18 & 10 & 32 \\
\hline Total & & 56 & 19 & 75 \\
\hline
\end{tabular}

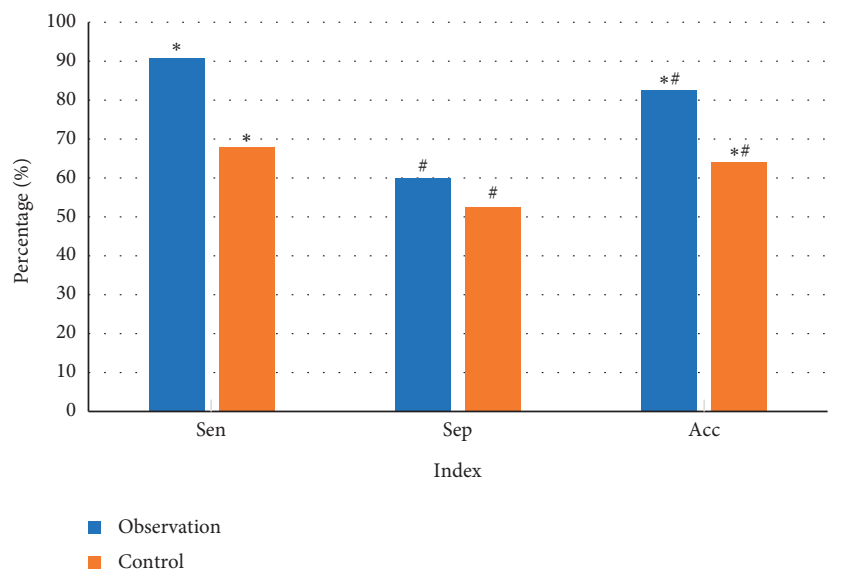

FiguRE 6: Comparison in consistency of comprehensive diagnosis results. *, \#, and * \# denote that the sensitivity, specificity, and accuracy of the two groups were statistically significant $(P<0.05)$. 
TABLe 5: The relationship between CAS and myocardial ischemia.

\begin{tabular}{lccc}
\hline \multirow{2}{*}{ Stenosis or not } & \multicolumn{2}{c}{ Comprehensive diagnosis } & Total \\
& Positive & Negative & \\
\hline Yes & 123 & 5 & 128 \\
No & 16 & 6 & 22 \\
Total & 139 & 11 & 150 \\
\hline
\end{tabular}

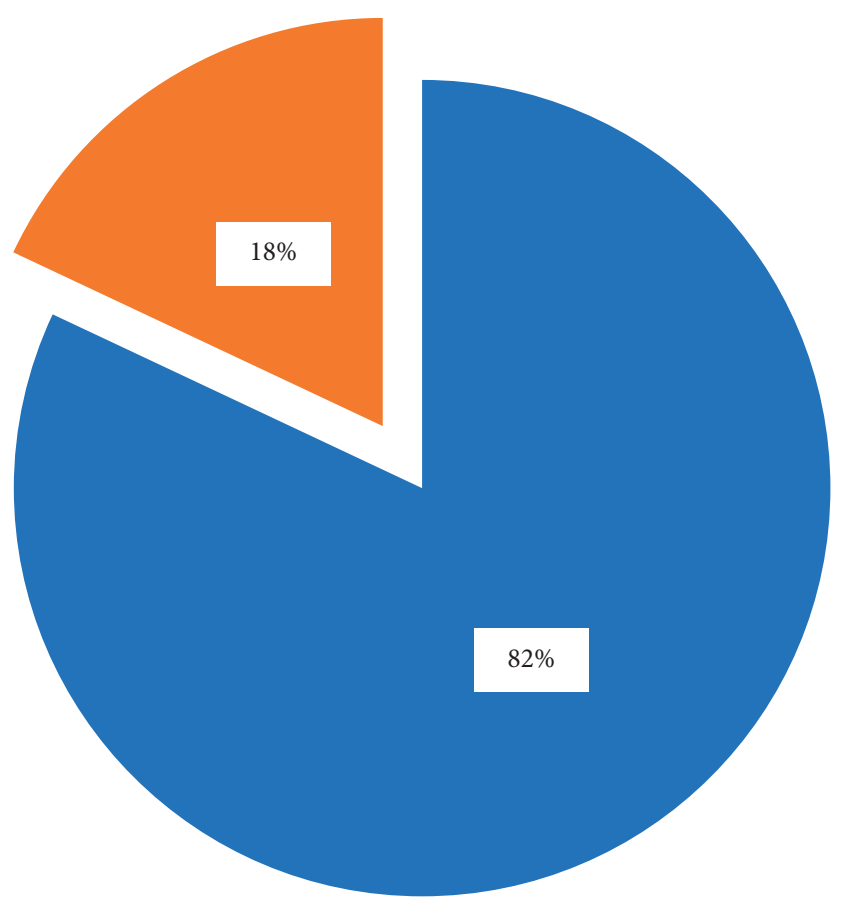

Positive\&Narrow

Others

FiguRe 7: Comparison on proportions.

clinical diagnosis of myocardial ischemia. The results showed that the sensitivity, specificity, and accuracy of the examination results of the observation group and the control group were $90.9 \%$ vs $67.8 \%, 60 \%$ vs $52.6 \%$, and $82.7 \%$ vs $64 \%$, respectively. It was obvious that the sensitivity, specificity, and accuracy of the observation group were higher than those of the control group, and the Kappa value of the observation group (0.72) was also higher than that of the control group (0.67), suggesting that the examination effect of the observation group was better than that of the control group. Some researchers have used neural network algorithms related to deep learning technology to create artificial intelligence models for CAD image vessel segmentation and vessel segment recognition and conducted practical research on $12900 \mathrm{CAD}$ images. The results showed that the accuracy of this method for automatic segmentation of CAD images of blood vessels reaches $99.2 \%$, and the average accuracy of segment recognition is $98.6 \%$. This shows that the advanced method can more accurately perform blood vessel segmentation and has a certain practicability, which can provide a reference for the examination and diagnosis of coronary vascular diseases in medical treatment [23]. The above research and the results of this study all indicated that deep learning had a good additive effect on CAD technology. The depth learning algorithm can be used in coronary angiography.

\section{Conclusion}

Based on the above analysis, CAD based on deep learning can more effectively diagnose patients with $\mathrm{CHD}$ accompanied by myocardial ischemia and can better show the lesions of the coronary arteries, suggesting the good development prospects of deep learning technology in medical imaging. However, there are some errors in this study due to the simplification of the research samples and the inadequacy of the research index setting, so that the inevitable connection among CAS, CHD, and myocardial ischemia was not proved better, and the relationship between them and other factors cannot be well proved, which will be improved in future studies.

\section{Data Availability}

The data used to support the findings of this study are available from the corresponding author upon request.

\section{Conflicts of Interest}

The authors declare no conflicts of interest.

\section{References}

[1] B. R. Pagliaro, F. Cannata, G. G. Stefanini, and L. Bolognese, "Myocardial ischemia and coronary disease in heart failure," Heart Failure Reviews, vol. 25, no. 1, pp. 53-65, 2020.

[2] H. Rahman, M. Ryan, M. Lumley et al., "Coronary microvascular dysfunction is associated with myocardial ischemia and abnormal coronary perfusion during exercise," Circulation, vol. 140, no. 22, pp. 1805-1816, 2019.

[3] M. Marzilli, F. Crea, D. Morrone et al., "Myocardial ischemia: from disease to syndrome," International Journal of Cardiology, vol. 314, pp. 32-35, 2020.

[4] V. Vaccarino, S. Sullivan, M. Hammadah et al., "Mental stress-induced-myocardial ischemia in young patients with recent myocardial infarction: sex differences and mechanisms," Circulation, vol. 137, no. 8, pp. 794-805, 2018.

[5] D. Lucini and M. Pagani, "Heart rate variability, autonomic regulation and myocardial ischemia," International Journal of Cardiology, vol. 312, pp. 22-23, 2020.

[6] K. Moazzami, S. Sullivan, B. B. Lima et al., "Mental stressinduced myocardial ischemia and cognitive impairment in coronary atherosclerosis," Journal of Psychosomatic Research, vol. 141, Article ID 110342, 2021.

[7] C. Collet, M. J. Grundeken, T. Asano, Y. Onuma, W. Wijns, and P. W. Serruys, "State of the art: coronary angiography," EuroIntervention, vol. 13, no. 6, pp. 634-643, 2017.

[8] R. Nakanishi, S. Motoyama, J. Leipsic, and M. J. Budoff, "How accurate is atherosclerosis imaging by coronary computed tomography angiography?" J Cardiovasc Comput Tomogr, vol. 13, no. 5, pp. 254-260, 2019.

[9] A. Hideo-Kajita, H. M. Garcia-Garcia, E. Shlofmitz, and C. M. Campos, "Update on coronary angiography-based 
physiology technologies," Arquivos Brasileiros de Cardiologia, vol. 113, no. 2, pp. 282-285, 2019.

[10] X. Zhu, Z. Cheng, S. Wang, X. Chen, and G. Lu, "Coronary angiography image segmentation based on PSPNet," Computer Methods and Programs in Biomedicine, vol. 200, Article ID 105897, 2021.

[11] J. Cui, H. Guo, H. Wang, F. Chen, L. Shu, and L. C. Li, "Fullyautomatic segmentation of coronary artery using growing algorithm," Journal of X-Ray Science and Technology, vol. 28, no. 6, pp. 1171-1186, 2020.

[12] D. Liang, J. Qiu, L. Wang et al., "Coronary angiography video segmentation method for assisting cardiovascular disease interventional treatment," BMC Medical Imaging, vol. 20, no. 1, p. $65,2020$.

[13] C. Guo, J. Lu, Z. Tian, W. Guo, and A. Darvishan, "Optimization of critical parameters of PEM fuel cell using TLBODE based on Elman neural network," Energy Conversion and Management, vol. 183, no. 3, pp. 149-158, 2019.

[14] M. Zhai, T. Du, R. Yang, and H. Zhang, "Coronary artery vascular segmentation on limited data via pseudo-precise label," in Proceedings of the 2019 41st Annual International Conference of the IEEE Engineering in Medicine and Biology Society (EMBC), pp. 816-819, Berlin Germany, July 2019.

[15] Y. Chen, S. Hu, H. Mao, W. Deng, and X. Gao, "Application of the best evacuation model of deep learning in the design of public structures," Image and Vision Computing, vol. 102, pp. 533-540, Article ID 103975, 2020.

[16] E. Pardo, J. M. T. Morgado, and N. Malpica, "Semantic segmentation of mFISH images using convolutional networks," Cytometry, Part A, vol. 93, no. 6, pp. 620-627, 2018.

[17] E. Shelhamer, J. Long, and T. Darrell, "Fully convolutional networks for semantic segmentation," IEEE Transactions on Pattern Analysis and Machine Intelligence, vol. 39, no. 4, pp. 640-651, 2017.

[18] Z. Wang, L. Xie, and J. Qi, "Dynamic pixel-wise weightingbased fully convolutional neural networks for left ventricle segmentation in short-axis MRI," Magnetic Resonance in Imaging, vol. 66, pp. 131-140, 2020.

[19] C. Richard Conti, "Myocardial ischemia is not always due to epicardial atheromatous disease," Clinical Cardiology, vol. 34, no. 1, pp. 8-9, 2011 Jan.

[20] J. A. Panza, T. A. Holly, F. M. Asch et al., "Inducible myocardial ischemia and outcomes in patients with coronary artery disease and left ventricular dysfunction," Journal of the American College of Cardiology, vol. 61, no. 18, pp. 1860-1870, 2013.

[21] M. Noël, J. Jobin, P. Poirier, G. R. Dagenais, and P. Bogaty, "Different thresholds of myocardial ischemia in ramp and standard bruce protocol exercise tests in patients with positive exercise stress tests and angiographically demonstrated coronary arterial narrowing," The American Journal of Cardiology, vol. 99, no. 7, pp. 921-924, 2007.

[22] T. Du, L. Xie, H. Zhang et al., "Training and validation of a deep learning architecture for the automatic analysis of coronary angiography," EuroIntervention, vol. 17, no. 1, pp. 32-40, 2021.

[23] J. M. Wolterink, T. Leiner, B. D. D. Vos, R. W. V. Hamersvelt, M. A. Viergever, and I. Išgum, "Automatic coronary artery calcium scoring in cardiac CT angiography using paired convolutional neural networks," Medical Image Analysis, vol. 34, pp. 123-136, 2016. 